\title{
Construcción y validación de una escala para evaluar salud sexual y reproductiva en adolescentes mujeres de la Región Caribe Colombiana.*
}

\author{
The Development and Validation of a scale to measure Reproductive and \\ Sexual Health of young women in the Colombian Caribbean region.
}

Recibido: 18 de mayo de 2015 | Aceptado: 17 de septiembre de 2015

doi:10.11144/Javeriana.upsy15-1.cvee

Para citar este artículo: Bello-Villanueva, A., Palacio, J., Vera-Villaroel, P., Rodríguez-Díaz, M., CelisAtenas, K., Pavez, P. (2016). Construcción y validación de una escala para evaluar salud sexual y reproductiva en adolescentes mujeres de la Región Caribe Colombiana. Universitas Psychologica, 15(1), 99-116. http://dx.doi.org/Javeriana.upsy15-1.cvee

Articulo de Investigacion. Esta investigación fue financiada por Colciencias como parte del proyecto código No. 121549326168, denominado: "Validación de estrategias pedagógicas y comunicacionales mediante un modelo cognitivo social y epidemiológico para la prevención de comportamientos sexuales de riesgo, en adolescentes escolarizados entre 11-16 años de dos ciudades del Caribe colombiano" junto con el apoyo de la Dirección de Investigación, Innovación y Desarrollo, Universidad del Norte, Barranquilla-Colombia.

Agradecimientos: La autora principal desea agradecer la asesoría, en la construcción del cuestionario Sexualidad Reproductiva (CSR), a la Dra. Elvia Vargas Trujillo tutora durante la pasantía en el Grupo.

** Grupo de investigaciones en Desarrollo Humano (GIDHUM), Departamento de Psicología, Universidad del Norte, Barranquilla, Colombia. Correspondencia: Ana Mercedes Bello Villanueva, Universidad del Norte, A.A. 1569-15820 Barranquilla- Atlántico, Colombia.E-mail: bellom@ uninorte.edu.co

**** Grupo de investigaciones en Desarrollo Humano (GIDHUM), Departamento de Psicología, Universidad del Norte, Barranquilla, Colombia.

****** Escuela de Psicología, Universidad de Santiago de Chile, Usach, Chile.

******* Centre for Accident Research and Road SafetyQueensland (CARRS-Q), Institute of Health and Biomedical Innovation (IHBI), Faculty of Health, Queensland University of Technology, Victoria Park Rd, Kelvin Grove, Queensland (QUT) 4059, Australia. Departamento de Ingeniería Industrial, Universidad del Norte, Barranquilla, Colombia.
Ana Mercedes Bello-Villanueva, Ph.D. **

Jorge Palacio, Ph.D. ***

Universidad del Norte, Colombia

Pablo Vera-Villarroel, Ph.D. *****

Universidad de Santiago de Chile, Chile

Oscar OviEdo-Trespalacios, M.S. ******

Queensland University of Technology (QUT), Australia

Universidad del Norte

Melissa Alejandra Rodríguez-Díaz, M.S.

Universidad del Norte, Barranquilla

Karem Celis-Atenas, Ph.D.

Paula Pavez

Universidad de Santiago de Chile, Chile

\section{RES U MEN}

El presente estudio tuvo como objetivo el diseño y validez de una escala para evaluar salud sexual y reproductiva. Participaron 919 estudiantes mujeres entre 11 a 19 años quienes respondieron el cuestionario, que evalúa actitudes y normas subjetivas, basado en planteamientos de la teoría de comportamiento planeado. Con el objetivo de comprobar la fiabilidad y validez del instrumento, se realizó un análisis factorial exploratorio y un análisis de componentes principales con rotación VARIMAX. Esta investigación incluye dos estudios independientes, en el estudio 1 (prueba piloto) la versión del pilotaje inicialmente constaba de 172 ítems y disminuyó a 78 en la muestra final. Para el estudio 2, en la escala de actitud se reporta una media de 3.68 (D.E. $=1.21$ ) y un $\alpha=0.91$. Se extrajeron dos factores agrupados en dos dimensiones que explican un $67.10 \%$; en la escala de normas subjetivas se obtuvo media de 3.80 (D.E. $=1.10$ ) y un $\alpha=0.97$. Los ítems se adecuan a la matriz factorial. Se extrajeron 11 factores de los cuales son interpretables 9 y se explican en un $75.89 \%$. En conclusión, el instrumento confirma una confiabilidad adecuada y estabilidad estructural de gran utilidad para la comprensión e implementación de estrategias de acción en sexualidad reproductiva en mujeres adolescentes.

Palabras clave

Creencias normativas; actitudes; sexualidad; mujeres adolescentes

\section{A B S T R ACT}

This article describes the design and validation of a scale for evaluating reproductive and sexual health. 919 young females from 11 to 19 years in five Caribbean-Colombian cities were administered the questionnaire that assesses attitudes and subjective norms about the reproductive sexuality, 
based on the planned behavior theory (Ajzen \& Fishbein, 2000). The reliability and validity of the instrument was analyzed through exploratory factorial analysis with principal components and VARIMAX rotation. The methodology includes two independent studies; in study 1 (pilot test) the first questionnaire consisted of 172 items and decreased to 78 in the final sample. For study 2, the attitude scale reported $($ Mean $=3.68, \mathrm{ED}=1.21)$ and Cronbach's alpha reliability was 0.91 . Two factors were extracted and grouped into two dimensions that explain $67.10 \%$ of the variance. For the subjective norms scale (Mean $=3.8, \mathrm{ED}=1.1$ ) Cronbach's alpha reliability was 0.97 and eleven factors were extracted and grouped into nine dimensions, which explain $75.89 \%$ of the variance. The scale showed good reliability and structural stability for the analysis and design of programs for sexual and reproductive health in adolescent girls.

Keywords

Normative beliefs; attitudes; sexuality; reproductive; adolescent females.

\section{Introducción}

En las últimas décadas, el incremento en las tasas de embarazos no planeados a temprana edad y abortos inducidos en la población de mujeres adolescentes (Flórez \& Soto, 2006; International Planned Parenthood Federation, 2010; Maddaleno, Morello, \& Infante, 2003) ha generado una preocupación a nivel nacional y mundial por estudiar y comprender las causas de estos fenómenos y por implementar intervenciones dirigidas a mejorar la salud sexual y el bienestar general de las jóvenes (Bauman, Karasz, \& Hamilton, 2007; Bello, Gómez, Mosquera, \& Palacio, 2010; Gayet \& Solís, 2007; L'Engle, Jackson, \& Brown, 2006; Méndez, Rodríguez, Lantigua, Acosta, \& Matos, 2007; Mosquera, Jiménez, \& Sanjuan, 2004)

En la región Caribe Colombiana se ha encontrado estudios que señalan una alta prevalencia de las relaciones sexuales en adolescentes entre los 13 y 17 años: un $25 \%$ y un $43.5 \%$ de los encuestados con edades menores y/o iguales a 13 años manifestaron haber iniciado su vida sexual (Campo-Arias, 2005). Así mismo, investigaciones más recientes reportan un notable aumento en las cifras de las jóvenes que realizan el debut sexual en un $42.9 \%$ antes de los 15 años de edad (Bello, Gómez et al., 2010; Vega, 2009), problemática que requiere de mayor atención y consideración como prioridad en salud pública en el litoral Caribe, debido a las altas tasas de muertes maternas, incidencia de las ITS y VIH/SIDA e incremento de las tasas de embarazo temprano no planeado y abortos (International Planned Parenthood Federation, 2010).

Diversos modelos teóricos han sido aplicados para identificar los antecedentes de estos comportamientos sexuales y reproductivos. Dentro de las teorías más utilizadas se encuentran la Teoría de la Acción Razonada - TAR (Fishbein \& Ajzen, 1975; Gilmore et al., 2002; Manoj \& Kanekar, 2007) y la Teoría del Comportamiento Planeado (Ajzen, 1991; Ajzen \& Manstead, 2010; Glasman \& Albarracín, 2003; Jemmott et al., 2007; Wise, Goggin, Gerkovich, Metcalf, \& Kennedy, 2006), las cuales han resultado exitosas en el diseño de proyectos de promoción de la salud dado su potencial para explicar los comportamientos saludables y de riesgo (Londoño \& Valencia, 2004; Prochaska $\&$ DiClemente, 1992; Tremblay \& Frigon, 2004).

La actitud es definida como la disposición a responder consistentemente de una manera favorable o desfavorable frente a un objeto; representa el resultado de la evaluación que el individuo realiza de dicho objeto, el cual es valorado en las dimensiones de bueno-malo, beneficioso- dañino, agradabledesagradable, placentero-no placentero, entre otras (Ajzen, 2001; Ajzen \& Fishbein, 2000). De acuerdo con el valor positivo o negativo asignado por la persona a las creencias, la actitud tendrá una dirección positiva o negativa también (Ajzen \& Sexton, 1999).

En este mismo sentido, la actitud se presenta en función de las creencias, referidas a las consecuencias percibidas por ejecutar el comportamiento y a la valoración que este individuo realiza de dichas consecuencias (Ajzen \& Fishbein, 1980, 2005). Se considera que existe la posibilidad de tener un número considerable de creencias acerca de un comportamiento especifico, sin embargo ciertas creencias determinarán la actitud personal (Conner \& Sparks, 1996; Flórez, 2007).

Las evidencias empíricas existentes señalan que la actitud personal hacia las relaciones sexuales en mujeres adolescentes latinas no es un factor predictor de la intención de tener relaciones (Flores, 
Tschann, \& Marin, 2002). En contraposición, se encuentran estudios que aseguran la existencia de una estrecha asociación entre las actitudes hacia las relaciones sexuales y la actividad sexual: los hallazgos sugieren que las mujeres adolescentes sexualmente activas tienen actitudes más permisivas frente a las relaciones sexuales pre-matrimoniales que quienes reportan no haber iniciado su actividad sexual (Aneshensel, Fielder, \& Becerra, 1989).

Por otro lado, se ha investigado la relación existente entre las actitudes y el comportamiento sexual de los jóvenes y los resultados afirman que las actitudes personales de los adolescentes, junto con la percepción de las actitudes de las personas cercanas y significativas hacia el inicio de la actividad sexual, influyen sobre la disposición y toma de decisión a tener relaciones sexuales en las y en los adolescentes (Bearman \& Brückner, 1999; Carvajal et al., 1999; Vargas \& Henao, 2007; Whitaker, Miller, \& Clark, 2000) así como en las prácticas de riesgo en su comportamiento sexual (Arrivillaga, Salazar, \& Correa, 2003; Fontanilla, Bello, \& Palacio, 2011).

Diversos estudios asociados a la norma subjetiva y social coinciden y apoyan la afirmación sobre la influencia de las personas cercanas y significativas en el comportamiento sexual de las mujeres adolescentes (Miller, McCoy, Olson, \& Wallace, 1986; Miller, Forehand, \& Kotchick, 1999) e identifican la importancia de los padres, adultos y amigos en el comportamiento sexual de las jóvenes.

La sociedad permanentemente evalúa y mide lo que, según sus normas, es permisible para hombres y mujeres en relación con la sexualidad. Se ha encontrado que culturalmente las conductas sexuales femeninas difieren de las masculinas en cuanto a la permisividad de conductas sexuales aceptables para hombres y de mayor restricción para las mujeres. (Sierra, Rojas, Ortega, \& Martín Ortiz, 2007 y Crawford \& Popp, 2003, como se citan en Rodríguez \& Toro, 2011). De esta misma forma los resultados sugieren que las diferencias de género en cuanto a las actitudes sexuales son notablemente significativas (Moral de la Rubia, 2011; Perla, Sierra, Vallejo-Medina, \& Gutiérrez Quintanilla, 2009) y requieren ser evaluadas de forma independiente (Hendrick's Sexual Attitudes Scale (Hendrick \&
Hendrick, 1987; Hendrick, Hendrick, \& Reich, 2006, citado en Petersen \& Hyde, 2010).

En relación con lo anterior, se ha encontrado en algunas investigaciones que las mujeres tienden a subestimar su experiencia sexual para ajustarse a las normas y presiones socioculturales (Alexander \& Fisher, 2003). Así mismo, otros estudios asociados al sistema de evaluación (Fisher, 2009; Jonason \& Fisher, 2009, como se citan en Petersen \& Hyde, 2010), también sugieren la necesidad de elaborar cuestionarios de autoinforme independientes, debido a que ha sido difícil interpretar el significado de tales diferencias de género debido a la confusión entre actitudes y creencias en las situaciones planteadas. Igualmente, argumentan la necesidad de incluir un mayor número de variables para el establecimiento y validación de modelos transculturales (Castro, Bermudez, Buela-Casal, \& Madrid, 2011; Ortega, Sánchez, Ortega-Rivera, Nocentini \& Menesini, 2010, como se cita en Sierra, Perla, \& Santos-Iglesia, 2011).

En las diferentes escalas internacionales y nacionales revisadas: Sexual attitudes. The 21-item Sexual Opinion Survey (Fisher, Byrne, \& White, 1983); Attitudes Toward Sexuality Scale (Fisher \& Hall, 1988; Barrera, Sarmiento, \& Vargas, 2004), no se contempla la evaluación de actitudes y creencias en formatos independientes, por esta razón, en este estudio se diseñó la escala de actitud y de percepción que tienen las mujeres adolescentes sobre la actitud de sus padres, profesores y amigos hacia la actividad sexual en la adolescencia en cuanto a comportamientos de prevención de riesgos sexuales.

En resumen, diversas investigaciones ponen en evidencia la gran influencia de las actitudes y percepciones en la actividad sexual en las mujeres adolescentes y del importante papel que cumplen en la educación sexual de las jóvenes (Bello, Mosquera, Palacio, \& Peralta, 2010; Fontanilla, et al., 2011).

La presente investigación tuvo dos objetivos: (1) analizar y valorar aspectos de administración de la prueba, comprensión de los ítems, tiempo de duración, etc., para determinar y conseguir una apreciación inicial de la confiabilidad, a través del análisis de consistencia interna Alfa de Cronbach y (2) obtener indicadores definitivos de su confiabi- 
lidad y validez. Ambos objetivos serán tratados en el documento como estudio 1 (objetivo 1) y estudio 2 (objetivo).

\section{Estudio 1: prueba piloto}

\section{Método}

\section{Participantes.}

En este estudio participaron 50 mujeres adolescentes de la región Caribe entre los 11 y 19 años de edad $(\mathrm{M}=14.38$ y D.E. $=1.77)$, su selección se hizo aleatoriamente en cada uno de los grados escolares entre $6^{\circ}$ a $11^{\circ}$. Pertenecientes a un nivel socioeconómico medio-bajo de una institución educativa pública en la ciudad de Barranquilla, firmaron consentimiento informado junto con sus padres $\mathrm{y} / \mathrm{o}$ acudientes.

Variables de estudio.

Se han considerado en dos grupos las variables de estudio, correspondientes a la escala de actitud en el primer grupo y las agrupadas en el segundo, en la escala de creencias, denominadas como normas subjetivas:

1. Las actitudes: se definen como una predisposición aprendida para responder de forma consistente, favorable o desfavorablemente con respecto a un objeto dado; la creencia sobre los objetos actitudinales se forma mediante la asociación de dicho objeto con determinados atributos (Ajzen, 2001). Abarca tres elementos fundamentales: actitud como consistencia, como predisposición a la respuesta y como disposición aprendida (Ajzen \& Fishbein, 1975).

Esta investigación entenderá las actitudes de acuerdo con la definición de Ajzen \& Fishbein (1975), como la evaluación que el individuo hace favorable o desfavorablemente hacia la actividad sexual y el comportamiento de protección reproductiva mediante adjetivos bipolares (necesario, saludable, maduro, seguro, responsable, fácil).

2. Creencias (normas subjetivas): de acuerdo con la teoría de comportamiento planeado existen tres tipos de creencias: conductuales, normativas y de control (Madden, Scholder, \& Ajzen, 1992). Las creencias normativas están dadas en función de las creencias que las jóvenes tienen acerca de cuál es la opinión de "otras personas o grupos de referencia importantes para ellas (padres, profesores y amigos), con respecto a si debe realizar o no el comportamiento sexual y reproductivo (Ajzen, 2001).

En el presente estudio se hizo énfasis en las creencias relacionadas con las normas subjetivas teniendo en cuenta que la opinión de referentes importantes sobre la realización de determinada conducta influye en la norma subjetiva. Se consideraron tres dimensiones (aprobación y apoyo, disposición a complacer e importancia de personas significativas).

Las creencias normativas influyen sobre la norma subjetiva percibida provocando cambios en dicho comportamiento (Martín, 2005). Están caracterizadas como factor social que se refiere a la presión social percibida para realizar o no el comportamiento (Ajzen, 2001), reflejan la influencia que el entorno inmediato al sujeto ejerce sobre su conducta.

\section{Instrumento}

El cuestionario estuvo constituido por 172 ítems en un formato de escala Likert con valores de 1 a 5 que indicaban el grado de acuerdo y/o desacuerdo frente a sus actitudes y creencias, desde 1 (Totalmente en desacuerdo) hasta 5 (Totalmente de Acuerdo).

\section{Procedimiento.}

El proceso de construcción del instrumento en la versión del pilotaje para la evaluación de actitudes y creencias (normas subjetivas) sobre la sexualidad reproductiva en adolescentes mujeres se describe en las siguientes fases:

Fase 1.

Se identificaron las dimensiones de evaluación contempladas en el instrumento, para lo que se 
revisó la literatura pertinente y actualizada, además se le consultó a jueces expertos en el tema y a sujetos que cumplían con las características de la muestra.

\section{Fase 2.}

Se determinó la construcción del instrumento a partir de investigaciones anteriores (Bello, Altamar, Mosquera, \& Palacio, 2010; Bello, Gómez et al., 2010; Bello Mosquera et al., 2010) basadas en el cuestionario llamado (ANCIP), diseñado por el grupo de investigadores, con base en los constructos teóricos especificados por la teoría de comportamiento planeado (Ajzen \& Fishbein, 2000). El instrumento tuvo como objetivo identificar actitudes y creencias en adolescentes tanto varones como mujeres. Se realizó previamente un pilotaje de las creencias personales, de control y grupos de referencia, mediante entrevistas cognitivas y encuestas estructuradas, para medir con los mismos parámetros del contexto al grupo seleccionado para la muestra.

La validez del constructo se realizó a través de acuerdo inter jueces. Una vez definido este nivel de acuerdo se eligieron únicamente aquellos ítems que tuvieran validez de contenido muestral. El instrumento original ANCIP diseñado constó de 110 ítems conformados por 40 reactivos sobre actitudes en relación con la evaluación de las prácticas de protección y de los resultados y también por 70 ítems de creencias normativas y de control percibido.

\section{Fase 3.}

En esta fase se procedió a la aplicación de la prueba piloto con un total de 172 ítems. Siguiendo las recomendaciones de las evaluaciones de expertos, se realizaron los cuestionarios para la versión femenina y masculina de manera independiente, después de seleccionar los ítems con validez de contenido muestral. Tanto el instrumento original como el cuestionario final fueron aprobados para la aplicación por el comité de Ética de la Universidad del Norte.

\section{Resultados Estudio 1: análisis muestra piloto}

Mediante el coeficiente Alfa de Cronbach se estimó la confiabilidad para el total de la escala de actitud, resultando un $\alpha=0.95$ y un $\alpha=0.98$ para la escala total de creencias (normas subjetivas). Posteriormente se descartaron aquellos ítems que no aportaban a la confiabilidad de la escala, es decir, 54 reactivos para las actitudes y 60 para creencias normativas. Una vez realizado este procedimiento, se estimó nuevamente la confiabilidad, arrojando el Alfa de Cronbach 0.91 para la escala total de actitud y un Alfa de 0.96 para la escala de normas subjetivas.

En relación con los ítems de actitud, se agruparon en tres dimensiones: actitud protección, actitud abstinencia y actitud retardo en el inicio de relaciones sexuales. En la primera, se reportó un alfa de 0.98 y se extrajeron 4 factores explicados en $82.68 \%$; en el segundo grupo de ítems, con un alfa de 0.95 se extrajeron 5 factores que se explican en $79.56 \%$ y finalmente, en el tercer grupo el alfa obtenido fue de 0.95 y se extrajeron 5 factores que se explican en un $76.30 \%$.

En cuanto a los ítems de creencias (normas subjetivas). El análisis de consistencia interna reporta en la variable normas subjetivas en cada una de sus dimensiones un Alfa de Cronbach de 0.84 en la dimensión de aprobación y apoyo padres; de 0.81 para aprobación y apoyo profesores y de 0.92 en la dimensión aprobación y apoyo amigos. En la disposición a complacer a otros en protección (pastillas orales, $\mathrm{AO}$ y pastillas de emergencia $\mathrm{AE}$ ), se registra alfa de 0.96 y en la disposición a complacer abstinencia un alfa de 0.94 y de 0.92 en disposición a complacer en retardo del inicio de la relación sexual. En la dimensión de importancia padres un alfa de 0.81 , seguido de un alfa de Cronbach de 0.82 en la importancia profesores en sexualidad reproductiva y de 0.83 en el grupo de ítems relacionados con la importancia amigos.

En el análisis de los componentes principales en las creencias normativas se observa que en cada una de las dimensiones de aprobación y apoyo (padres, profesores y amigos) se extraen 2 factores explicados en un $84.95 \%, 86.82 \%$ y $81.79 \%$ respectivamen- 
te. En la dimensión de disposición a complacer se explican porcentualmente: protección (87.39), abstinencia (80.27) y retardo (71.16) y se extrae un factor para cada dimensión; y en la dimensión de importancia padres, profesores y amigos se observan dos componentes extraídos en cada grupo de ítems, que se explican en un $75.59 \%, 79.60 \%$ y en un $80.25 \%$ respectivamente.

\section{Estudio 2: prueba final}

\section{Método}

\section{Participantes estudio 2: prueba final.}

En este estudio se seleccionaron 868 mujeres de la región Caribe, entre 11 y 19 años de edad, $(\mathrm{M}=14.26$; D.E. $=1.98$. Pertenecientes a los grados de escolaridad de $6^{\circ}$ a $11^{\circ}$, con nivel socioeconómico medio-bajo, vinculadas a 15 instituciones educativas en 5 ciudades de la región Caribe colombiana. El número de estudiantes entre $6^{\circ}$ a $7^{\circ}$ fue de 262 (29.8\%), en los grados $8^{\circ}$ a $9^{\circ}$ fue de $305(34.6 \%)$ y de $10^{\circ}$ a $11^{\circ}$ fue de 310 (35.6\%).

TABLA 1.

Variables Socio-demográficas de la muestra $(n=879)$.
A través de muestreo por conglomerados se consideró la representatividad de las 5 ciudades en cada uno de los departamentos de la región Caribe, Los criterios de exclusión e inclusión para seleccionar la muestra fueron: estudiantes entre 11 y 19 años, de instituciones con desempeño medio en los exámenes de estado ICFES en el 2009, para incrementar un equilibrio académico, matriculados en grados de $6^{\circ}$ a $11^{\circ}$ en la jornada mañana o tarde en colegios mixtos. En la muestra seleccionada en el estudio se excluyeron los colegios nocturnos.

Los participantes con previa autorización de sus padres y/o acudientes firmaron consentimiento informado que les garantizaba confidencialidad utilizando código clave de identificación, teniendo en consideración las normas éticas emanadas por el Ministerio de Protección Social en Colombia y por la American Psychological Association 2002 (APA).

\section{Variables demográficas.}

Están comprendidas en la información recopilada con respecto a la edad, grado de escolaridad que cursan los jóvenes y ciudad de estudio. (Véase tabla 1)

\begin{tabular}{cc}
\hline Característica & No. (\%) \\
\hline Edad (años) & $14.27(1.98)$ \\
Media (DE) & 316 \\
$10-13$ & 451 \\
$14-16$ & 109 \\
$17-19$ & \\
Grado Escolar & $8.53(1.72)$ \\
Media (DE) & 262 \\
$6-7$ & 305 \\
$8-9$ & 310 \\
$10-11$ & 220 \\
Ciudad & 266 \\
Barranquilla & 130 \\
Santa Marta & 182 \\
Cartagena & 81 \\
Montería & \\
Riohacha & \\
\hline
\end{tabular}

Fuente: elaboración propia 


\section{Variables de estudio.}

Las variables del estudio 2 son similares a las descritas en el estudio 1. Corresponden a las actitudes (protección y abstinencia) y creencias normativas de los adolescentes en relación con su comportamiento sexual y reproductivo.

\section{Procedimiento}

\section{Fase 1.}

Se realizó una revisión de los resultados estructurales del cuestionario en el estudio 1 (muestra piloto) para realizar ajustes pertinentes y posteriormente evaluación de jueces expertos nacionales e internacionales. De esta forma, la prueba final quedó establecida en una escala de 78 ítems tipo Likert, frente a la que los sujetos debían responder entre 5 alternativas de respuestas que iban desde la afirmación nada saludable hasta muy saludable, nada seguro hasta muy seguro, nada importante hasta muy importante y desde muy en desacuerdo hasta muy de acuerdo. Las afirmaciones podían indicar dirección favorable o positiva y/o desfavorable o negativa.

\section{Fase 2.}

Aplicación definitiva del instrumento, finalmente la prueba se administró a 929 participantes mujeres adolescentes, a fin de obtener indicadores definitivos de su confiabilidad y validez. Un grupo de 30 encuestadores distribuidos en las 5 ciudades administraron la prueba final durante un tiempo promedio de 45 minutos por encuestador.

\section{Análisis de Datos.}

Se realizó el análisis de los datos de la valoración de las escalas a través del Paquete Estadístico para Ciencias Sociales (IBM SPSS), versión 21.0 para Windows. El análisis de la consistencia interna se realizó calculando el coeficiente alfa de Cronbach y la evaluación de la validez de constructo se ejecutó por medio del análisis factorial exploratorio: análisis de componentes principales con rotación VARIMAX.

\section{Resultados Estudio 2: Análisis muestra definitiva}

\author{
Análisis fiabilidad. \\ Escala de actitud.
}

La media obtenida en la escala de actitud fue de 3.68 (D.E.= 1.21), el alfa de Cronbach obtenido para esta escala fue de 0.91, la evaluación de la homogeneidad fue elevada en todos los casos obteniéndose correlación ítem total superiores a 0.50. La estimación de la consistencia interna para cada una de las dimensiones fue de 0.93 en la dimensión actitud hacia la protección con pastillas orales $(\mathrm{AO})$ y pastillas de emergencia (AE) y de 0.93 en la actitud abstinencia/retardo (ver tabla 2).

\section{Escala de normas subjetivas}

Para la escala de normas subjetivas se obtuvo una media de 3.80 (D.E.= 1.10), la estimación de la confiabilidad por medio de alfa de Cronbach fue de 0.94 La estimación de la consistencia interna para cada una de las dimensiones fue de 0.92 en aprobación/apoyo padres, abstinencia/retardo y de 0.93 en aprobación/apoyo profesores y amigos abstinencia/retardo, mientras que en la dimensión aprobación/apoyo protección (AO, AE) fue de 0.95 para padres, profesores y amigos; para la dimensión disposición a complacer a padres, profesores y amigos en protección (AO, AE) al igual que en la de disposición a complacer padres, profesores y amigos en abstinencia/retardo se obtuvo un alfa de 0.95. Finalmente para la dimensión de Importancia padres, profesores y amigos en protección fue de 0.93 , mientras que en la dimensión de Importancia padres, profesores y amigos en abstinencia/retardo fue de 0.94; la homogeneidad de la prueba fue adecuada con correlaciones superiores a 0.40 . En tabla 3 a y 3 b se detalla media, desviación estándar, correlación ítem total y alfa de Cronbach para cada una de las dimensiones del cuestionario (Aprobación y Apoyo; Disposición a complacer; Importancia). 
TABLA 2.

Media, Desviación Estándar, Correlación ítem total Escala de Actitud

\begin{tabular}{lccc}
\hline \multicolumn{1}{c}{ Escala } & Media & D.E. & $r$ Item-total \\
\hline \multicolumn{1}{c}{ Actitud protección } & & & \\
Usar AO evitar embarazo es RESPONSABLE & 4.05 & 1.20 & $0.74^{* *}$ \\
Usar AE evitar embarazo es RESPONSABLE & 4.15 & 1.07 & $0.76^{* *}$ \\
Usar AE evitar embarazo es NECESARIO & 4.18 & 1.02 & $0.73^{* *}$ \\
Usar AO evitar embarazo es SALUDABLE & 3.99 & 1.08 & $0.76^{* *}$ \\
Usar AE evitar embarazo es SALUDABLE & 4.15 & 1.04 & $0.77^{* *}$ \\
Usar AO evitar embarazo es MADURO & 4.04 & 1.05 & $0.76^{* *}$ \\
Usar AE evitar embarazo es MADURO & 4.13 & 1.04 & $0.77^{* *}$ \\
Usar AO evitar embarazo es SEGURO & 4.03 & 1.03 & $0.78^{* *}$ \\
Usar AE evitar embarazo es SEGURO & 4.14 & 1.05 & $0.76^{* *}$ \\
$\quad$ Actitud abstinencia/retardo & & & \\
Posponer relaciones 18 años ITS SALUDABLE & 3.53 & 1.31 & $0.70^{* *}$ \\
Posponer relaciones 18 años embarazo MADURO & 3.60 & 1.23 & $0.60^{* *}$ \\
Posponer relaciones 18 años ITS SEGURO & 3.67 & 1.26 & $0.60^{* *}$ \\
Evitar relaciones adolescencia embarazo MADURO & 3.59 & 1.20 & $0.62^{* *}$ \\
Evitar relaciones adolescencia ITS MADURO & 3.55 & 1.28 & $0.72^{* *}$ \\
Evitar relaciones adolescencia embarazo SEGURO & 3.69 & 1.20 & $0.71^{* *}$ \\
Evitar relaciones adolescencia ITS SEGURO & 3.57 & 1.33 & $0.71^{* *}$ \\
\hline
\end{tabular}

Nota. $* * p<0.01$

Fuente: elaboración propia

TABLA 3A.

Media, Desviación Estándar, Correlación ítem total y Cronbach Escala de Normas Subjetivas

\begin{tabular}{lcccc}
\hline \multicolumn{1}{c}{ Escala } & Media & D.E. & rItem-total & (alfa de Cronbach) \\
\hline \multicolumn{1}{c}{ Dimensión Aprobación/Apoyo } & & & & \\
Padres aprobarían usar AO embarazo & 3.33 & 1.322 & $0.570^{* *}$ & 0.967 \\
Padres aprobarían usar AE embarazo & 3.19 & 1.293 & $0.589^{* *}$ & 0.967 \\
Padres apoyarían usar AO embarazo & 3.35 & 1.253 & $0.634^{* *}$ & 0.967 \\
Padres apoyarían usar AE embarazo & 3.15 & 1.274 & $0.598^{* *}$ & 0.967 \\
Padres acuerdo usar AO embarazo & 3.07 & 1.352 & $0.550^{* *}$ & 0.967 \\
Padres acuerdo usar AE embarazo & 3.01 & 1.323 & $0.541 * *$ & 0.967 \\
Profesores aprobarían usar AO embarazo & 3.47 & 1.162 & $0.631 * *$ & 0.967 \\
Profesores aprobarían usar AE embarazo & 3.32 & 1.177 & $0.616^{* *}$ & 0.967 \\
Profesores apoyarían usar AO embarazo & 3.47 & 1.136 & $0.650^{* *}$ & 0.967 \\
Profesores apoyarían usar AE embarazo & 3.32 & 1.188 & $0.630^{* *}$ & 0.967 \\
Profesores acuerdo usar AO embarazo & 3.32 & 1.239 & $0.604 * *$ & 0.967 \\
Profesores acuerdo usar AE embarazo & 3.23 & 1.235 & $0.601 * *$ & 0.967 \\
Padres aprobarían evitar adoles. embarazo & 4.39 & 0.973 & $0.367 * *$ & 0.967 \\
Padres aprobarían evitar adoles. ITS & 4.42 & 0.952 & $0.402^{* *}$ & 0.967 \\
Padres aprobarían posponer 18 embarazo & 4.19 & 1.057 & $0.473 * *$ & 0.967 \\
Padres aprobarían posponer 18 ITS & 4.20 & 1.107 & $0.460 * *$ & 0.967 \\
Padres apoyarían evitar adoles. embarazo & 4.38 & 0.965 & $0.454^{* *}$ & 0.967 \\
Padres apoyarían evitar adoles. ITS & 4.43 & 0.951 & $0.431^{* *}$ & 0.967
\end{tabular}




\begin{tabular}{lcccc}
\hline \multicolumn{1}{c}{ Escala } & Media & D.E. & rItem-total & (alfa de Cronbach) \\
\hline Profesores aprobarían evitar adoles. ITS & 4.23 & 0.934 & $0.483^{* *}$ & 0.967 \\
Profesores aprobarían posponer18 embarz & 4.09 & 0.997 & $0.592^{* *}$ & 0.967 \\
Profesores aprobarían posponer18 ITS & 4.13 & 0.989 & $0.599^{* *}$ & 0.967 \\
Profesores apoyarían evitar adoles embarz & 4.18 & 0.986 & $0.523^{* *}$ & 0.967 \\
Profesores apoyarían posponer 18 embarz & 4.06 & 1.016 & $0.576^{* *}$ & 0.967 \\
Amigos aprobarían evitar adoles. ITS & 3.89 & 1.002 & $0.453^{* *}$ & 0.967 \\
Amigos aprobarían posponer 18 embarazo & 3.69 & 1.061 & $0.555^{* *}$ & 0.967 \\
Amigos aprobarían posponer18 ITS & 3.80 & 1.071 & $0.543^{* *}$ & 0.967 \\
Amigos apoyarían evitar adoles. Embarz & 3.88 & 0.997 & $0.443^{* *}$ & 0.967 \\
Amigos apoyarían evitar adoles. ITS & 3.95 & 1.012 & $0.476^{* *}$ & 0.967 \\
Amigos apoyarían posponer 18 embarazo & 3.78 & 1.040 & $0.600^{* *}$ & 0.967 \\
Amigos aprobarían usar AO embarazo & 3.58 & 1.138 & $0.606^{* *}$ & 0.967 \\
Amigos aprobarían usar AE embarazo & 3.51 & 1.164 & $0.617^{* *}$ & 0.967 \\
Amigos apoyarían usar AO embarazo & 3.55 & 1.157 & $0.624^{* *}$ & 0.967 \\
Amigos apoyarían usar AE embarazo & 3.48 & 1.180 & $0.595^{* *}$ & 0.967 \\
Amigos acuerdo usar AO embarazo & 3.54 & 1.145 & $0.585^{* *}$ & 0.967 \\
Amigos acuerdo usar AE embarazo & 3.50 & 1.185 & $0.572^{* *}$ & 0.967 \\
\hline
\end{tabular}

Nota. $* * p<0.01$

Fuente: Elaboración propia

TABLA 3B.

Media, Desviación Estándar, Correlación ítem total, alfa Escala de Normas Subjetivas

\begin{tabular}{|c|c|c|c|c|}
\hline Escala & Media & D.E. & rItem-total & (alfa de Cronbach) \\
\hline \multicolumn{5}{|l|}{ Dimensión Disposición complacer } \\
\hline Dispuesto complacer padres posponer 18 embarazo & 4.01 & 1.112 & $0.615 * *$ & 0.967 \\
\hline Dispuesto complacer padres posponer 18 ITS & 4.12 & 1.096 & $0.605^{* *}$ & 0.967 \\
\hline Dispuesto complacer profesores posponer 18 embarazo & 3.91 & 1.061 & $0.630 * *$ & 0.967 \\
\hline Dispuesto complacer amigos posponer 18 embarazo & 3.88 & 1.076 & $0.626 * *$ & 0.967 \\
\hline Dispuesto complacer amigos posponer 18 ITS & 4.01 & 1.065 & $0.611 * *$ & 0.967 \\
\hline Dispuesto complacer padres evitar embarazo & 4.20 & 1.012 & $0.526 * *$ & 0.967 \\
\hline Dispuesto complacer profesores evitar embarazo & 4.07 & 0.976 & $0.540 * *$ & 0.967 \\
\hline Dispuesto complacer padres $\mathrm{AO}$ embarazo & 3.62 & 1.295 & $0.636 * *$ & 0.967 \\
\hline Dispuesto complacer profesores $\mathrm{AO}$ embarazo & 3.55 & 1.239 & $0.623 * *$ & 0.967 \\
\hline Dispuesto complacer profesores AE embarazo & 3.42 & 1.298 & $0.605 * *$ & 0.967 \\
\hline Dispuesto complacer amigos $\mathrm{AO}$ embarazo & 3.53 & 1.287 & $0.623 * *$ & 0.967 \\
\hline Dispuesto complacer amigos AE embarazo & 3.42 & 1.310 & $0.585 * *$ & 0.967 \\
\hline \multicolumn{5}{|l|}{ Dimensión Importancia } \\
\hline Importante Padres posponer 18 embarazo & 4.25 & 0.957 & $0.535 * *$ & 0.967 \\
\hline Importante padres posponer 18 años ITS & 4.30 & 0.942 & $0.545 * *$ & 0.967 \\
\hline Importante padres evitar ITS & 4.49 & 0.820 & $0.478 * *$ & 0.967 \\
\hline Importante padres $\mathrm{AO}$ evitar embarazo & 4.00 & 1.107 & $0.561 * *$ & 0.967 \\
\hline Importante padres $\mathrm{AE}$ evitar embarazo & 3.89 & 1.116 & $0.563 * *$ & 0.967 \\
\hline Importante profesores $\mathrm{AO}$ evitar embarazo & 3.80 & 1.124 & $0.580 * *$ & 0.967 \\
\hline Importante profesores $\mathrm{AE}$ evitar embarazo & 3.70 & 1.162 & $0.573 * *$ & 0.967 \\
\hline Importante profesores posponer 18 embarazo & 4.01 & 1.005 & $0.560 * *$ & 0.967 \\
\hline Importante profesores evitar embarazo & 4.11 & 0.940 & $0.472 * *$ & 0.967 \\
\hline
\end{tabular}


Ana Mercedes Bello-Villanueva, Jorge Palacio, Pablo Vera-Villarroel, Oscar OviedoTrespalacios, Melissa Alejandra Rodríguez-Díaz, Karem Celis-Atenas, Paula Pavez

\begin{tabular}{lllll}
\hline \multicolumn{1}{c}{ Escala } & Media & D.E. & rItem-total & (alfa de Cronbach) \\
\cline { 1 - 2 } Importante profesores evitar ITS & 4.18 & 0.903 & $0.504^{* *}$ & 0.967 \\
Importante amigos posponer 18 embarazo & 3.94 & 1.085 & $0.552^{* *}$ & 0.967 \\
Importante amigos posponer 18 ITS & 4.07 & 1.071 & $0.545^{* *}$ & 0.967 \\
Importante amigos evitar embarazo & 4.05 & 1.012 & $0.494^{* *}$ & 0.967 \\
Importante amigos AO embarazo & 3.68 & 1.115 & $0.578^{* *}$ & 0.967 \\
Importante amigos AE embarazo & 3.64 & 1.127 & $0.576^{* *}$ & 0.967 \\
\hline
\end{tabular}

Nota. $* * \mathrm{p}<0.01$

Fuente: elaboración propia

\section{Análisis estructura factorial.}

\section{Escala de actitud}

La evaluación de la validez de constructo se realizó por medio del análisis factorial exploratorio. Se realizó análisis de componentes principales con rotación VARIMAX. La adecuación de los datos a la matriz factorial fue satisfactoria, se encontró un $\mathrm{KMO}=$ 0.876 y la prueba de esfericidad de Bartlett fue alta- mente significativa $\left(\mathrm{Chi}^{2}=10100 ; \mathrm{gl}=120 ; p<0.01\right)$. Se extrajeron 2 factores que explican un $67.10 \%$ de la varianza, la estructura factorial se representa por 16 ítems escalonados en dos grupos: el primer factor lo integran 9 ítems relacionados con la dimensión actitud protección mediante ( $\mathrm{AO}$ y $\mathrm{AE}$ ) y el segundo factor lo conforman un grupo de 7 reactivos asociados a la dimensión actitud hacia la abstinencia y retardo en el inicio de la relaciones sexuales, en tabla 4 se observa el detalle de la solución factorial.

TABLA 4.

Análisis Factorial Exploratorio por Componentes Principales y Rotación Varimax - Escala Actitud

\begin{tabular}{|c|c|c|}
\hline Ítems & $\begin{array}{c}\text { Factor } \\
1\end{array}$ & $\begin{array}{c}\text { Factor } \\
2\end{array}$ \\
\hline \multicolumn{3}{|l|}{ Dimensión Actitud Protección } \\
\hline Usar AO evitar embarazo es RESPONSABLE & 0.65 & \\
\hline Usar AE evitar embarazo es RESPONSABLE & 0.66 & \\
\hline Usar AE evitar embarazo es NECESARIO & 0.75 & \\
\hline Usar AO evitar embarazo es SALUDABLE & 0.8 & \\
\hline Usar AE evitar embarazo es SALUDABLE & 0.85 & \\
\hline Usar AO evitar embarazo es MADURO & 0.79 & \\
\hline Usar AE evitar embarazo es MADURO & 0.86 & \\
\hline Usar AO evitar embarazo es SEGURO & 0.81 & \\
\hline Usar AE evitar embarazo es SEGURO & 0.83 & \\
\hline \multicolumn{3}{|l|}{ Dimensión Actitud Abstinencia/Retardo } \\
\hline Posponer relaciones 18 años ITS SALUDABLE & & 0.77 \\
\hline Posponer relaciones 18 años embarazo MADURO & & 0.79 \\
\hline Posponer relaciones 18 años ITS SEGURO & & 0.79 \\
\hline Evitar relaciones adolescencia embarazo MADURO & & 0.84 \\
\hline Evitar relaciones adolescencia ITS MADURO & & 0.85 \\
\hline Evitar relaciones adolescencia embarazo SEGURO & & 0.86 \\
\hline Evitar relaciones adolescencia ITS SEGURO & & 0.87 \\
\hline
\end{tabular}

Nota. Varianza total explicada: $67,10 \%$

Fuente: elaboración propia 


\section{Escala normas subjetivas}

La valoración de la dimensionalidad de la escala se realizó por medio del análisis factorial exploratorio por componentes principales y rotación VARIMAX. El conjunto de 62 ítems se adecuada a la matriz factorial, encontrándose un $\mathrm{KMO}=0.946$ y altamente significativa la prueba de esfericidad de Bartlett $\left(\mathrm{Chi}^{2}=51917,053 ; \mathrm{gl}=1891 ; p<0.01\right)$. Se extrajeron 11 factores de los cuales son interpretables 9 y se explican en un $75.89 \%$ de la varianza total, En tabla 5 a y 5 b se detalla solución factorial.

\section{TABLA 5A.}

Análisis Factorial Exploratorio por Componentes Principales y Rotación Varimax - Escala Normas Subjetivas

\begin{tabular}{|c|c|c|c|c|c|c|c|c|c|}
\hline Ítems & F1 & $\mathrm{F} 2$ & F3 & F4 & F5 & F6 & F7 & F8 & F9 \\
\hline \multicolumn{10}{|l|}{ Dimensión Aprobación/Apoyo Adultos Protección } \\
\hline Padres aprobarían usar $\mathrm{AO}$ embarazo & 0.8 & & & & & & & & \\
\hline Padres aprobarían usar AE embarazo & 0.8 & & & & & & & & \\
\hline Padres apoyarían usar AO embarazo & 0.8 & & & & & & & & \\
\hline Padres apoyarían usar AE embarazo & 0.9 & & & & & & & & \\
\hline Padres acuerdo que jóvenes usen $\mathrm{AO}$ embarazo & 0.8 & & & & & & & & \\
\hline Padres acuerdo que jóvenes usen AE embarazo & 0.8 & & & & & & & & \\
\hline Profesores aprobarían usar $\mathrm{AO}$ embarazo & 0.7 & & & & & & & & \\
\hline Profesores aprobarían usar AE embarazo & 0.7 & & & & & & & & \\
\hline Profesores apoyarían usar $\mathrm{AO}$ embarazo & 0.7 & & & & & & & & \\
\hline Profesores apoyarían usar AE embarazo & 0.7 & & & & & & & & \\
\hline Profesores acuerdo que jóvenes usen $\mathrm{AO}$ embarazo & 0.5 & & & & & & & & \\
\hline Profesores acuerdo que jóvenes usen AE embarazo & 0.5 & & & & & & & & \\
\hline \multicolumn{10}{|l|}{ Dimensión Aprobación/Apoyo Amigos Protección } \\
\hline Amigos aprobarían usar $\mathrm{AO}$ embarazo & & 0.8 & & & & & & & \\
\hline Amigos aprobarían usar AE embarazo & & 0.8 & & & & & & & \\
\hline Amigos apoyarían usar $\mathrm{AO}$ embarazo & & 0.8 & & & & & & & \\
\hline Amigos apoyarían usar AE embarazo & & 0.8 & & & & & & & \\
\hline Amigos de acuerdo que jóvenes usen $\mathrm{AO}$ embarazo & & 0.8 & & & & & & & \\
\hline Amigos de acuerdo que jóvenes usen AE embarazo & & 0.8 & & & & & & & \\
\hline \multicolumn{10}{|l|}{ Dimensión Aprobación/Apoyo Padres Abstinencia/Retardo } \\
\hline Padres aprobarían evitar relaciones adolescencia embarazo & & & 0.8 & & & & & & \\
\hline Padres aprobarían evitar relaciones adolescencia ITS & & & 0.8 & & & & & & \\
\hline Padres aprobarían posponer relaciones 18 años embarazo & & & 0.7 & & & & & & \\
\hline Padres aprobarían posponer relaciones 18 años ITS & & & 0.7 & & & & & & \\
\hline Padres apoyarían evitar relaciones adolescencia embarazo & & & 0.8 & & & & & & \\
\hline Padres apoyarían evitar relaciones adolescencia ITS & & & 0.79 & & & & & & \\
\hline
\end{tabular}

Fuente:elaboración propia

Tabla $5 b$.

Análisis Factorial Exploratorio por Componentes Principales y Rotación Varimax - Escala Normas Subjetivas

$\begin{array}{llllllllll}\text { Ítems } & \text { F1 } & \text { F2 } & \text { F3 } & \text { F4 } & \text { F5 } & \text { F6 } & \text { F7 } & \text { F8 } & \text { F9 }\end{array}$

Dimensión Aprobación/Apoyo Profesores abstinencia/Retardo

Profesores aprobarían evitar relaciones adolescencia ITS 0.6

$\begin{array}{ll}\text { Profesores aprobarían posponer relaciones } 18 \text { años embarazo } & 0.7\end{array}$

Profesores aprobarían posponer relaciones 18 años ITS $\quad 0.7$ 
Ana Mercedes Bello-Villanueva, Jorge Palacio, Pablo Vera-Villarroel, Oscar OviedoTrespalacios, Melissa Alejandra Rodríguez-Díaz, Karem Celis-Atenas, Paula Pavez

\begin{tabular}{|c|c|c|c|c|c|c|c|c|c|}
\hline Ítems & F1 & F2 & F3 & F4 & F5 & F6 & F7 & F8 & F9 \\
\hline Profesores apoyarían evitar relaciones adolescencia embarazo & & & & 0.5 & & & & & \\
\hline Profesores apoyarían posponer relaciones 18 años embarazo & & & & 0.6 & & & & & \\
\hline \multicolumn{10}{|l|}{ Dimensión Aprobación/Apoyo Amigos Abstinencia/Retardo } \\
\hline Amigos aprobarían evitar relaciones adolescencia ITS & & & & & 0.8 & & & & \\
\hline Amigos aprobarían posponer relaciones 18 años embarazo & & & & & 0.7 & & & & \\
\hline Amigos aprobarían posponer relaciones 18 años ITS & & & & & 0.8 & & & & \\
\hline Amigos apoyarían evitar relaciones adolescencia embarazo & & & & & 0.8 & & & & \\
\hline Amigos apoyarían evitar relaciones adolescencia ITS & & & & & 0.8 & & & & \\
\hline Amigos apoyarían posponer relaciones 18 años embarazo & & & & & 0.7 & & & & \\
\hline \multicolumn{10}{|l|}{$\begin{array}{c}\text { Dimensión Disposición complacer/todas personas Protección } \\
\text { (AE, AO) }\end{array}$} \\
\hline Dispuesto complacer padres usar $\mathrm{AO}$ embarazo & & & & & & 0.7 & & & \\
\hline Dispuesto complacer profesores usar $\mathrm{AO}$ embarazo & & & & & & 0.7 & & & \\
\hline Dispuesto complacer profesores usar AE embarazo & & & & & & 0.7 & & & \\
\hline Dispuesto complacer amigos usar $\mathrm{AO}$ embarazo & & & & & & 0.7 & & & \\
\hline Dispuesto complacer amigos usar AE embarazo & & & & & & 0.7 & & & \\
\hline \multicolumn{10}{|l|}{$\begin{array}{c}\text { Dimensión Disposición a complacer/todas personas } \\
\text { Abstinencia/Retardo }\end{array}$} \\
\hline Dispuesto complacer padres evitar relaciones embarazo & & & & & & & 0.7 & & \\
\hline Dispuesto complacer padres posponer 18 años embarazo & & & & & & & 0.8 & & \\
\hline Dispuesto complacer padres posponer 18 años ITS & & & & & & & 0.7 & & \\
\hline Dispuesto complacer profesores evitar relaciones embarazo & & & & & & & 0.7 & & \\
\hline Dispuesto complacer profesores posponer 18 años embarazo & & & & & & & 0.8 & & \\
\hline Dispuesto complacer amigos posponer 18 años embarazo & & & & & & & 0.8 & & \\
\hline Dispuesto complacer amigos posponer 18 años ITS & & & & & & & 0.7 & & \\
\hline \multicolumn{10}{|l|}{ Dimensión Importancia todas personas $(\mathrm{AO}, \mathrm{AE})$} \\
\hline Importante padres piensen $\mathrm{AO}$ embarazo & & & & & & & & 0.7 & \\
\hline Importante padres piensen $\mathrm{AE}$ embarazo & & & & & & & & 0.8 & \\
\hline Importante profesores piensen $\mathrm{AO}$ embarazo & & & & & & & & 0.8 & \\
\hline Importante profesores piensen $\mathrm{AE}$ embarazo & & & & & & & & 0.8 & \\
\hline Importante amigos piensen $\mathrm{AO}$ embarazo & & & & & & & & 0.7 & \\
\hline Importante amigos piensen $\mathrm{AE}$ embarazo & & & & & & & & 0.8 & \\
\hline \multicolumn{10}{|l|}{ Dimensión Importancia todas personas Abstinencia/Retardo } \\
\hline Importante Padres piensen posponer 18 años embarazo & & & & & & & & & 0.7 \\
\hline Importante padres piensen posponer 18 años ITS & & & & & & & & & 0.7 \\
\hline Importante padres piensen evitar relaciones ITS & & & & & & & & & 0.7 \\
\hline Importante profesores piensen posponer 18 años embarazo & & & & & & & & & 0.8 \\
\hline Importante profesores piensen evitar embarazo & & & & & & & & & 0.8 \\
\hline Importante profesores piensen evitar relaciones ITS & & & & & & & & & 0.7 \\
\hline Importante amigos piensen posponer 18 años embarazo & & & & & & & & & 0.8 \\
\hline Importante amigos piensen posponer 18 años ITS & & & & & & & & & 0.8 \\
\hline Importante amigos piensen evitar relaciones embarazo & & & & & & & & & 0.8 \\
\hline
\end{tabular}

Nota. Varianza total explicada: $75.89 \%$

Fuente: elaboración propia 


\section{Discusión}

El propósito de esta investigación fue diseñar y validar un cuestionario de sexualidad reproductiva dirigido al género femenino, a través del análisis de la consistencia interna y validez del constructo en las dos escalas de actitud y creencias normativas, teniendo en cuenta que estas dos variables eran las de mayor predicción en la intenciones comportamentales (Fishbein \& Ajzen, 1975; BelloVillanueva, Palacio, Rodríguez-Díaz, \& OviedoTrespalacios, 2013).

Para las ciencias sociales los resultados obtenidos en el análisis de fiabilidad y validez de la estructura factorial son satisfactorios en las escalas de actitud y de creencias normativas en el cuestionario de sexualidad reproductiva en adolescentes mujeres y confirman su adecuación y utilidad práctica como punto de partida para diagnosticar las actitudes normativas en el comportamiento sexual y reproductivo en las jóvenes escolares, basados en los criterios de análisis descritos en estudios previos (Hair, Black, Babin, \& Anderson, 2010; Sierra, Oviedo-Trespalacios, Candelo, \& Soto, 2014). Este resultado es análogo al obtenido previamente por Bello-Villanueva et al. (2013) en la versión para varones de la escala para evaluar actitudes y creencias sobre la sexualidad reproductiva en adolescentes de la región Caribe colombiana.

Así mismo, estas escalas facilitan el diseño e implementación de programas preventivos y de promoción específicos basados en líneas y estrategias de acción óptimas en la actividad sexual y reproductiva en el género femenino para la prevención de comportamientos de riesgos en este ámbito.

En cuanto a la escala de actitud, se obtuvieron dos factores: el primero dirigido a un grupo de 9 ítems conformados por la actitud hacia la protección con pastillas orales y/o pastillas de emergencia, en el segundo grupo se observaron 7 ítems relacionados con la abstinencia y retardo en el inicio de relaciones sexuales. Al parecer las jóvenes al momento de responder no logran comprender una diferencia clara en los ítems relacionados con la actitud abstinencia de los dirigidos al retardo en la iniciación de la actividad sexual y comparten las cargas factoriales entre los ítems, que puede explicarse por la semejanza en la interpretación del concepto por parte de las jóvenes adolescentes y por la similaridad en el constructo, el cual tiene la posibilidad de diferenciarse por la acción del tiempo en la utilización de estas medidas de prevención.

En la configuración factorial de la escala de normas subjetivas se aprecian 9 factores: en primer lugar la dimensionalidad de aprobación y apoyo que se agrupo en 5 factores (1 a 5) dirigidos a la protección y a la abstinencia/retardo, implica la percepción que ellas tienen sobre poder contar con el apoyo de otros y acerca de lo que piensan, opinan y aprueban en su contexto con respecto a la actividad sexual reproductiva y en este mismo sentido, abarca la percepción de presión e influencia social y familiar que tiene la joven en la necesidad de aprobación y búsqueda de apoyo y aceptación por parte de figuras importantes y significativas como padres, profesores y amigos; en segundo lugar se engloban dos factores ( 6 y 7 ) referenciados en los ítems asociados con la disposición a complacer a dichas personas significativas en protección mediante la utilización de $\mathrm{AO}$ y $\mathrm{AE}$ para prevenir embarazos e ITS, encierran la creencia relacionada con la disposición a complacer para obtener la aceptación y el reconocimiento de personas importantes en la vida de la joven estudiante; y en tercer lugar, se identificaron dos factores (8 y 9) correspondientes a la dimensión de importancia frente a los métodos, que incluyen la relevancia dada por las jóvenes adolescentes a lo que piensan sobre el uso de estrategias de protección, abstinencia y retardo en el inicio de las relaciones sexuales.

Los factores identificados en las dos escalas son similares a los hallazgos reportados en otros estudios que reportan evidencias empíricas de la relación existente entre las actitudes y creencias normativas en la sexualidad reproductiva de las adolescentes (Barrera, Sarmiento, \& Vargas, 2004; Cantillo, Escorcia, Escorcia, \& Herazo, 2007; Vargas \& Henao, 2007; Vega, 2009; Whitaker, et al., 2000).

En términos generales, puede concluirse que el cuestionario de sexualidad reproductiva representado en las dos escalas contribuye a generar una herramienta de gran utilidad psicométrica para la 
evaluación y diagnóstico de actitudes y creencias normativas en las jóvenes adolescentes en el contexto investigativo y educativo en esta población específica que promueva la elección de estrategias efectivas en la prevención de riesgos sexuales (International Planned Parenthood Federation, 2010; Vinaccia et al., 2007) así como la elección de comportamientos sexuales protectores (Pérez \& Pick, 2006; Tucker, Fitzmaurice, Imamura, \& Penfold, 2007) conductas de gran importancia, considerando el crecimiento desproporcionado de embarazos no planeados ni deseados en adolescentes de esta población, problemática contemplada como prioritaria en salud pública en Colombia (BelloVillanueva, Palacio, Rodríguez-Díaz, \& OviedoTrespalacios, 2015; Campo, 2009; Fontanilla et al., 2011; UNFPA, 2010).

\section{Referencias}

Ajzen, I. (1991). The theory of planned behavior. Organizational Behavior and Human Decission Processes, 50(2), 179-211.

Ajzen, I. (2001). Nature and operation of attitudes. Annual Review of Psychology, 52(1), 27-58.

Ajzen, I., \& Fishbein, M. (1980). Understanding attitudes and predicting social behavior. United States of America: Prentice Hall.

Ajzen, I., \& Fishbein, M. (2000). Attitudes and the attitude behavior relation: Reasoned and automatic processes. European Review of Social Psychology, 11(1), 1-33.

Ajzen, I., \& Fishbein, M. (2005). The influence of attitudes on behavior. In D. Albarracín, B. Johnson \& M. Zanna (Eds.), The handbook of attitudes (pp. 173-221 ). Mahwah, NJ: Erlbaum.

Ajzen, I., \& Manstead, A. (2010). Changing healthrelated behaviours. An approach based on the theory of planned behaviour. Recuperado el $11 \mathrm{de}$ agosto de 2003 de http://people.umass.edu/aizen/

Ajzen, I., \& Sexton, J. (1999). Depth of processing, belief congruence, and attitude-behavior correspondence. In S. Chaiken \& Y. Trope (Eds.), Dual-process Theories in Social Psychology (pp. 117-138). New York: Guilford.
Alexander, M. G. \& Fisher, T. D. (2003). Truth and Consequences: Using the Bogus Pipeline to Examine Sex Differences in Self-Reported Sexuality. The Journal of Sex Research, 40, (1), February 2003: pp 27-35.

Aneshensel, C., Fielder, E., \& Becerra, R. (1989). Fertility and fertility related behavior among Mexican.American and non-Hispanic White female adolescents. Journal of Health and Social Behavior, 30(1), 56-76.

Arrivillaga, M., Salazar, I., \& Correa, D. (2003). Creencias sobre la salud y su relación con las prácticas de riesgo o de protección en jóvenes universitarios. Revista Colombia Médica, 34, 186-195.

Barrera, F., Sarmiento, E., \& Vargas, E. (2004). Relación de las actitudes personales y de la norma social con la actividad sexual de los adolescentes. Revista de Estudios Sociales, 17, 56-66.

Bauman, L., Karasz, A., \& Hamilton, A. (2007). Understanding failure of condom use intention among adolescents: Completing an intensive preventive intervention. Journal of Adolescent Research, 22(3), 248-274. doi: 10.1177/0743558407299696

Bearman, P., \& Brückner, H. (1999). Power in Numbers: Peer effects on adolescent girls' sexual debut and pregnancy National Campaing to Prevent Teen Pregnancy. Washington, DC. National Campaign to Prevent Teen Pregnancy.

Bello, A., Altamar, N., Mosquera, M., \& Palacio, J. (2010). Actitud, norma subjetiva, control percibido e intención hacia las prácticas de protección sexual para la prevención de embarazos no planeados e infecciones de transmisión sexual ITS/VIH/SIDA en adolescentes escolarizados de la ciudad de Barranquilla. Tesis de Magíster en Psicología no publicada, Universidad del Norte, Barranquilla, Colombia.

Bello, A., Gómez, J., Mosquera, M., \& Palacio, J. (2010). Actitud, norma subjetiva, control percibido e intención hacia las prácticas de protección sexual para la prevención de embarazos no planeados ITS y VIH/ SIDA en adolescentes escolarizados de Ciénaga. Tesis de Magíster en Psicología no publicada, Universidad del Norte, Barranquilla, Colombia.

Bello, A., Mosquera, M., Palacio, J., \& Peralta, S. (2010). Actitud, norma subjetiva, control percibido e intención hacia las prácticas de protección sexual para la 
prevención de embarazos no planeados ITS y VIH/ SIDA en adolescentes escolarizados del municipio de Sincelejo. Tesis de Magíster en Psicología, Universidad del Norte, Barranquilla, Colombia.

Bello-Villanueva, A. M., Palacio, J., Rodríguez-Díaz, M., \& Oviedo-Trespalacios, O. (2013). Medición de la Intención en la Actividad Sexual en Adolescentes: una Aproximación de Acuerdo al Género del Caribe Colombiano. Terapia psicológica, 31(3), 343-353.

Bello-Villanueva, A. M., Palacio, J., Rodríguez-Díaz, M., \& Oviedo-Trespalacios, O. (2015). Correlación entre la personalidad y los factores de la Teoría del Comportamiento Planeado (TCP) en adolescentes escolarizados de 11-19 años del Caribe Colombiano. Terapia psicológica, 33(3), 169-180.

Bello-Villanueva, A. M., Oviedo-Trespalacios, O., VeraVillarroel, P., Oviedo, O., Rodríguez-Diaz, M., Celis-Atenas, K., \& Pavez, P. (2014). Presentación de una escala para evaluar actitudes y creencias sobre la sexualidad reproductiva en adolescentes varones de la región Caribe colombiana. Universitas Psychologica, 13(1). 47-60

Campo, A. (2009). Relaciones sexuales en adolescentes colombianos y las implicaciones para la salud pública: Una revisión de la prevalencia y algunas variables asociadas. MedUNAB, 12(2), 86-90.

Cantillo, Á., Escorcia, I., Escorcia, K., \& Herazo, K. (2007). Prácticas, creencias y actitudes de los docentes frente a la educación sexual, en escuelas básica primaria y secundaria en la ciudad de Barranquilla. Tesis de pregrado en Enfermería no publicada, Universidad del Norte, Barranquilla.

Carvajal, S., Parcel, G., Banspach, S., Basen-Enqquist, K., Coyle, K., Kirby, D., \& Chan, W. (1999). Psychosocial predictors of delay of first sexual intercourse by adolescents. Health Psychology, 18(5), 443-452.

Castro, A., Bermúdez, M., Buela-Casal, G., \& Madrid, J. (2011). Variables psicosociales que median en el debut sexual de adolescentes en España. Revista Latinoamericana de Psicología, 43(1), 83-94.

Campo-Arias, A. (2005). Prevalencia de uso de condón en la primera relación sexual en adolescentes de Santa Marta, Colombia: diferencias por género. Medunab, 8, 59-64.
Conner, M., \& Sparks, P. (1996). The theory of planned behaviour and health behaviours. Philadelphia: Open University Press.

Fishbein, M., \& Ajzen, I. (1975). Belief, Attitude, Intention, and Behavior: An Introduction to Theory and Research. Reading, MA: Addison-Wesley.

Fisher, W.A., Byrne, D., \& White, L.A, (1983). Emotional barriers to contraception in D. Byrne \& W.A. Fisher (Eds.), Adolescents, sex contraception. Hilldales, NJ: Lawrence Erlbaum.

Fisher, T.D. \& Hall, R.G. (1988). A scale for the comparison of the sexual attitudes of adolescents and their parents. Journal of Sex Research, 24, 90-100.

Flores, E., Tschann, J. M., \& Marin, B. V. (2002). Latina adolescents: Predicting intentions to have sex. Adolescence, 37(148), 659-679.

Flórez, A. (2007). Psicología social de la salud. Promoción y prevención. Bogotá: El Manual Moderno.

Flórez, C., \& Soto, V. (2006). Fecundidad adolescente y desigualdad en Colombia y la Región de América Latina y el Caribe. Trabajo presentado en la Reunión de Expertos sobre Población y Pobreza en América Latina y el Caribe Santiago, Chile.

Fontanilla, S., Bello, A., \& Palacio, J. (2011). Caracterización de actitudes, creencias y conocimiento en función de la intención de los comportamientos sexuales y reproductivos en los adolescentes escolarizados en la ciudad de Santa Marta. Magíster en Psicología, Universidad del Norte, Barranquilla.

Gayet, C., \& Solís, P. (2007). Sexualidad saludable de los adolescentes: La necesidad de políticas basadas en evidencias. Salud Pública de México, 49, 47-51.

Gilmore, M., Archibald, M., Morrison, D., Wilsdon, A., Wells, E., Hoppe, M.,. Murowchick, E. (2002). Teen sexual behavior: Applicability of the theory of reasoned action. Journal of Marriage and Family 64(4), 885-897.

Glasman, L. R., \& Albarracín, D. (2003). Models of health - related behaviour: A study of condom use in two cities of Argentina. AIDS and Behaviour, 7, $183-193$.

Hair, J., Black, W., Babin, B., \& Anderson, R. (2010). Multivariate Data Analysis (6a. ed). Upper Saddle River, NJ: Prentice-Hall. 
Ana Mercedes Bello-Villanueva, Jorge Palacio, Pablo Vera-Villarroel, Oscar OviedoTrespalacios, Melissa Alejandra Rodríguez-Díaz, Karem Celis-Atenas, Paula Pavez

Hernández-Sampieri, R., Fernández-Collado, C., \& Baptista-Lucio, M. (2010). Metodología de la investigación. México: Mc Graw Hill.

International Planned Parenthood Federation. (2010). Datos sobre la salud sexual y reproductiva de mujeres adolescentes en el mundo en desarrollo Recuperado de http://www.guttmacher.org/pubs/ FB-Adolescents-SRH-SP.pdf

Jemmott, J. B., Heeren, G., Ngwane, Z., \& Hewitt, N., Jemmott, L., Shell, R. \& O'Leary, A. (2007). Theory of planned behaviour predictors of intention to use condoms among Xhosa adolescents in South Africa. AIDS Care, 19, 677-684.

L'Engle, K., Jackson, C., \& Brown, J. (2006). Early adolescents' cognitive susceptibility to initiating sexual intercourse. Perspective on Sexual and Reproductive Health, 38(2), 97-105.

Londoño, P. C., \& Valencia, L. C. (2004). Validez empírica de los modelos cognoscitivos sociales de prevención, Tesis de Magíster en Psicología, Universidad Nacional de Colombia, Bogotá.

Maddaleno, M., Morello, P., \& Infante, F. (2003). Salud y desarrollo de adolescentes y jóvenes en Latinoamérica y El Caribe: Desafíos para la próxima década. Salud Pública de México, 45(1), S132-S139.

Madden, T. J., Scholder, E. \& Ajzen, I. (1992). "A comparison of the theory of planed behavior and the theory of reasoned actino". Personality and Social Psychology Bulletin, 18(1), 3-9.

Manoj, S., \& Kanekar, A. (2007). Theory of reasoned action \& theory of planned behavior in alcohol and drug education. Journal of Alcohol and Drug Education, 51(1), 3-5.

Martín, M. (2005). Violencia juvenil exogrupal. Madrid: Eurydice.

Méndez, R., Rodríguez, E., Lantigua, L., Acosta, E., \& Matos, I. (2007). Frecuencia de la primera relación sexual en adolescentes de 12 - 18 años de edad en una Escuela Pública del sector de los Alcarrizos. Rev.Méd.Dom., 68(2), 186-189.

Miller, B., McCoy, J. K., Olson, T., \& Wallace, C. (1986). Parental discipline and control attempts in relation to adolescents sexual attitudes and behavior. Journal of Marriage and the Family, 48, 503-512.

Miller, K. S., Forehand, R., \& Kotchick, B. A. (1999). Adolescent sexual behavior in two ethnic minor- ity samples: The role of family variable. Journal of Marriage and the family, 61(1), 85-98.

Moral de la Rubia, J. (2011). Homosexualidad en la juventud mexicana y su distribución geográfica. Papeles de población, 17(67), 11-134.

Mosquera, M., Jiménez, E., \& Sanjuan, J. (2004). Evaluación y Planificación de un plan de comunicación para la prevención de embarazos en adolescentes en el contexto escolar [Proyecto]. Barranquilla: Fundación Universidad del Norte,

Pérez, C., \& Pick, S. (2006). Conducta sexual protegida en adolescentes mexicanos. Interamerican Journal of Psychology, 40(3), 333-340.

Perla, F., Sierra, J.C., Vallejo-Medina, P. \& Gutiérrez Quintanilla, R., (2009). Un estudio psicométrico de la versión española reducida del Hulbert Index of Sexual Fantasy. Boletín de Psicología, 96, 7-16.

Petersen, J., \& Hyde, J. (2010). A Meta- Analytic Review of Research on Gender Differences in sexuality, 1993-2007. Psychological Bulletin, 136(1), 21-38.

Prochaska, J., \& DiClemente, C. (1992). Stages of change in the modification of problem behaviors. In E. Herson, \& P.M. Miller (Eds.) Progress in Behavior Modification (pp.183-218). Sycamore, IL: Sycamore Publishing.

Rodríguez, Y. \& Toro, J. (2011). Resistencia al sexo: cultura y personalidad. Revista Interamericana de Psicologia, 45(1), 3-9.

Sierra, R. C., Oviedo-Trespalacios, O., Candelo, J. E., \& Soto, J. D. (2014). The influence of atmospheric conditions on the leakage current of ceramic insulators on the Colombian Caribbean coast. Environmental Science and Pollution Research, 1-11.

Sierra, J., Perla, F. \& Santos-Iglesia, P. (2011). Culpabilidad sexual en jóvenes: influencia de las actitudes y la experiencia sexual. Revista Latinoamericana de Psicología, 43(1), 73-81.

Tremblay, L., \& Frigon, J. Y. (2004). Biobehavioural and Cognitive Determinants of Adolescent Girls' Involvement in Sexual Risk Behaviours: A Test of Three Theoretical Models. The Canadian Journal of Human Sexuality, 13(1), 29-43.

Tucker, J., Fitzmaurice, A., Imamura, M., \& Penfold, S. (2007). The effect of the national demonstration project healthy respect on teenage sexual health 
behavior. European Journal of Public Health, 1, 33-39.

UNFPA, C. (2010). Población y equidad en Colombia. Análisis de situación. Volumen I. Recuperado de http://www.unfpacolombia.org/menuSup. php?id=5

Vargas, E., Henao, J., \& González, C. (2007). Toma de decisiones sexuales y reproductivas en la adolescencia. Acta Colombiana de Psicología, 10(1), 49-63.

Vega, J. (2009). Caracterización de las normas sociales, actitudes, habilidades para la vida, intenciones y comportamientos relacionados con la posibilidad de ocurrencia de embarazos en adolescentes. Estudio de línea base cuantitativa y cualitativa. (Informe resultados línea de base población adolescente 12-20 años) Barranquilla: Fundación Universidad del Norte. Vinaccia, S., Margarita, J., Gaviria, A., Soto, A., Gil, M., \& Ballester, R. (2007). Conductas sexuales de riesgo para la infección por Vih/Sida en Adolescentes colombianos. Terapia Psicológica, 25, 39-50.

Whitaker, D., Miller, K., \& Clark, L. (2000). Reconceptualizing adolescents sexual behavior: Beyond did they or didn't they? Family Planning Perspectives, 32(3), 111-117.

Wise, D., Goggin, K., Gerkovich, M., Metcalf, K., \& Kennedy, S. (2006). Predicting intentions to use condoms using gender, sexual experience, and the theory of planned behavior. American Journal of health Education, 37(4), 210-218. 
